Proc Indian Natn Sci Acad $\mathbf{8 1}$ No. 1 February 2015 Special Issue, pp. 40-50

(C) Printed in India.

DOI: $10.16943 /$ ptinsa/2015/v81il/48049

\title{
The Multiple Facets of Correlation Functions
}

\author{
CLAUDE PRUNEAU* \\ Physics and Astronomy Department, Wayne State University, 666 West Hancock, \\ Detroit, Michigan, 48152 USA
}

(Received on 21 May 2014; Accepted on 16 June 2014)

I will review studies of fluctuations and correlations carried out over the last 20 years and hopefully provide some insights for new measurements.

Key Words : QGP; Correlation; Charge Fluctuation; Momentum Fluctuation

\section{Introduction}

Correlation observables are a key component of the toolset used by researchers in the study of the dynamics of nuclear collisions at high energy, and for measurements of the properties of the matter produced in these collisions. As such, correlation observables take a multitude of forms and provide access to a broad range of phenomena and nuclear matter properties (Fig. 1). While they seemingly take many different forms, correlation observables share a basic definition and properties. They can be broadly divided into integral and differential correlation functions, which may be averaged over all interactions measured for a given type of collisions, or studied as function of global event observables such as the total transverse energy, or the charged particle multiplicity measured in a specific kinematic range. Differential correlations can be studied as function of selected kinematical variables of two or more particles and integral correlations are well suited towards the study of fluctuations. In these proceedings, I review advances in measurements of several types of correlation and fluctuation observables towards the identification and study of the quark gluon plasma (QGP) produced in relativistic heavy ion collisions at the CERN SPS, RHIC, and the LHC. I first introduce the basic definition of correlation observables in $\S$. I then proceed to review a number of measurements that were designed to identify and study the properties of the QGP, including net charge fluctuations in $\S$, the charge balance function in $\S$, higher moments of charge fluctuations in $\S$, measurements of $K / \pi$ yield

\footnotetext{
*Author for Correspondence : E-mail: pruneau@physics.waynne.edu
} 
fluctuations in $\S$, and $p_{T}$ fluctuations in $\S$. I conclude with a brief assessment of the results and impact of these various measurements in $\S$.

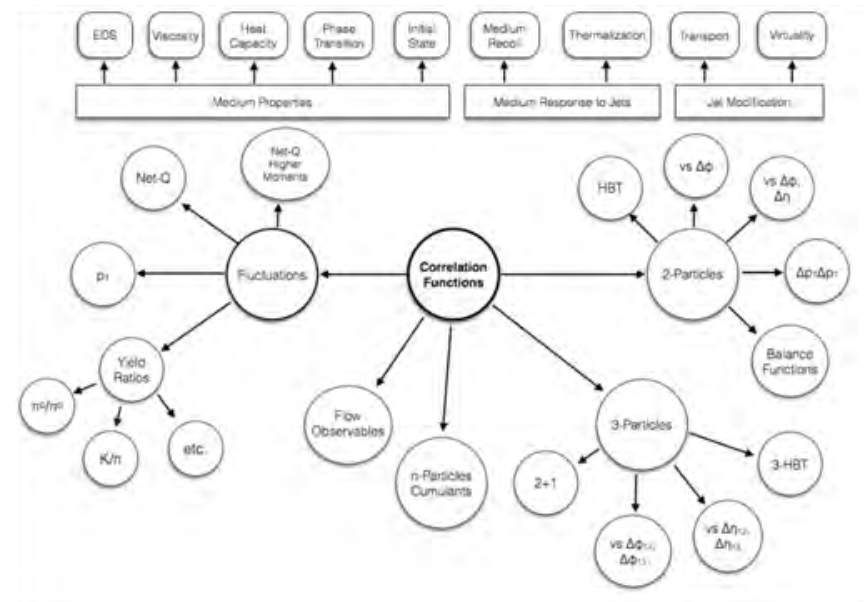

Fig. 1: The multiple facets of correlation functions: Differential and integral correlations have a common ground in terms of cumulants, and enable the definition of several types of correlation observables

\section{Definitions}

Measurements of correlations and fluctuations are rooted in the notion of joint probability distribution and multi-particle density. For instance, two random variables $x$ and $y$ can be said to be statistically independent if their joint probability distribution, $P(x, y)$, can be shown to factorize, i.e. if $P(x, y)=P(x) P(y)$ where $P(x)$ and $P(y)$ are the probabilities of observing $x$ and $y$ independently of each other. In the context of measurements of particle production in nuclear collisions, random variables of interest are the particle yield or particle density at different positions in momentum space. One is in particular interested in measuring whether the yield of particle production at a specific momentum (or range of coordinates) is correlated to the production at another momentum or range of coordinates. Loosely speaking, such a measurement can be accomplished by measuring the covariance of the two yields. But since particle densities are in fact functions of the momentum coordinates, one is led to the introduction of correlation functions which compare the two-particle density, $\rho_{2}\left(\vec{p}_{1}, \vec{p}_{2}\right)$, expressing the number of particle pairs at two specific momenta (or into two ranges) with the product of the single particle densities $\rho_{1}\left(\vec{p}_{1}\right)$ and $\rho_{1}\left(\vec{p}_{2}\right)$. Such correlation functions may be expressed as a difference or a ratio as follows:

$$
\begin{aligned}
C_{\alpha \beta}\left(\vec{p}_{\alpha}, \vec{p}_{\beta}\right) & =\rho_{2}\left(\vec{p}_{\alpha}, \vec{p}_{\beta}\right)-\rho_{1}\left(\vec{p}_{\alpha}\right) \times \rho_{1}\left(\vec{p}_{\beta}\right) \\
R_{\alpha \beta}\left(\vec{p}_{\alpha}, \vec{p}_{\beta}\right) & =\frac{C_{\alpha \beta}\left(\vec{p}_{\alpha}, \vec{p}_{\beta}\right)}{\rho_{1}\left(\vec{p}_{\alpha}\right) \times \rho_{1}\left(\vec{p}_{\beta}\right)}=\frac{\rho_{2}\left(\vec{p}_{\alpha}, \vec{p}_{\beta}\right)}{\rho_{1}\left(\vec{p}_{\alpha}\right) \times \rho_{1}\left(\vec{p}_{\beta}\right)}-1 .
\end{aligned}
$$


Generalization to three or more particles is readily achieved with the use of cumulants. Integration of single, two-, and $n$-particle densities over a finite momentum volume yield the average particle, $\langle N\rangle$, the average number of particle pairs, $\langle N(N-1)\rangle$, and factorial moments, $\langle N(N-1) \cdots(N-n)\rangle$ in that volume. Given the variance of the particle yield $\left\langle\Delta N^{2}\right\rangle=\left\langle N^{2}\right\rangle-\langle N\rangle^{2}$ may also be written $\left\langle\Delta N^{2}\right\rangle=$ $\langle N(N-1)\rangle-\langle N\rangle^{2}+\langle N\rangle$, one finds that integrals of the correlation functions $C_{2}$ or $R_{2}$ provide measures of particle fluctuations. Fluctuation observables based on $R_{2}$, in particular, offer the advantage that they are robust (i.e. independent) against particle loses associated with detection efficiencies, whereas $\left\langle\Delta N^{2}\right\rangle$ has a non trivial dependence on efficiencies.

\section{Net Charge Fluctuations}

In the late 1990s, early 2000s, Koch et al. (Jeon and Koch, 1999, 2000; Koch et al., 2002; Bleicher et al., 2000), Asakawa et al., (Asakawa et al., 2000), Heiselberg et al., (Heiselberg, 2001; Baym and Heiselberg 1999), and several others (Shuryak, 1998; Shuryak and Stephanov, 2011; Aziz and Gavin, 2004; Zhang et al., 2002; Bialas, 2002; Bopp and Ranft, 2001) elaborated on the notion that net charge fluctuations, i.e., fluctuations of the net charge of particles measured in a fixed momentum volume, might be severely suppressed if a quark gluon plasma phase is produced in the midst of heavy ion collisions, relative to fluctuations in proton-proton collisions, or a resonance gas. In particular, Koch et al., (Jeon and Koch, 2000) predicted the observable $D \equiv\left\langle N_{c h}\right\rangle\left\langle\Delta R^{2}\right\rangle$ would span different ranges of values for a free gas of particles $(D \approx 4)$, a resonance gas $(D \approx 3)$, and a quark gluon plasma $(D \approx 1)$. The observable $D$ then emerged as an essential tool to determine whether a QGP is in fact produced in relativistic heavy ion collisions, and several experimental collaborations pursued its measurement at the CERN SPS, at RHIC, and eventually at the LHC. The problem initially arose that different groups chose to carry out measurements of net charge fluctuations using different and seemingly incompatible observables. For instance, the NA49 collaboration reported measurements using the $\Phi_{q}$ observable, while PHENIX and STAR used $\omega \equiv\left\langle\Delta Q^{2}\right\rangle /\left\langle N_{c h}\right\rangle$ and $\nu_{+-, d y n}$ respectively (Alt et al., 2004; Adcox et al., 2002a; Adams et al., 2003a; Abelev et al., 2009a). Fortunately, there exist a relatively simple one-to-one relationships between these observables (Pruneau $e t$ al., 2003). The $\nu_{+-, d y n}$ observable involves the advantage of being defined in terms of ratios of two particle correlators to product of single particle yields, and is thus robust against particle losses due to detection efficiencies.

$$
\begin{aligned}
\nu_{+-, d y n} & =\frac{\left\langle N_{+}\left(N_{+}-1\right\rangle\right)}{\left\langle N_{+}\right\rangle^{2}}+\frac{\left\langle N_{-}\left(N_{-}-1\right)\right\rangle}{\left\langle N_{-}\right\rangle^{2}}-2 \frac{\left.\left\langle N_{+} N_{-}\right\rangle\right)}{\left\langle N_{+}\right\rangle\left\langle N_{-}\right\rangle} \\
& =R_{++}+R_{--}-2 R_{+-} .
\end{aligned}
$$

However, by construction, $\nu_{+-, d y n}$ is subject to a $1 / m$ scaling in the presence of $m$ independent particle sources. Measurements were thus reported both in terms of $\nu_{+-, d y n}$ and in terms of $N_{c h} \nu_{+-, d y n}$ where 
$N_{c h}$ is total number of charged particles measuring in the fiducial volume of experiments, corrected for detection efficiencies and instrumental effects. Fig. 2 presents measurements reported by STAR (Abelev et al., 2009a) for $\mathrm{Au}+\mathrm{Au}$ and $\mathrm{Cu}+\mathrm{Cu}$ collision systems at several beam energies as well as results by ALICE Abelev et al., 2013) for $\mathrm{Pb}+\mathrm{Pb}$ and $\mathrm{p}+\mathrm{p}$ collisions. One finds that $\nu_{+-, d y n}$ exhibits the anticipated $1 / m$ scaling behavior for all collision systems and energies considered. However, the quantity $N_{c h} \nu_{+-, d y n}$ exhibits a clear dependence on collision centrality which indicate a change in the strength of the charged particle production correlation in central collisions. Both the STAR and ALICE measurements indicate a relative suppression of the fluctuations in central collisions. The effect is weaker at RHIC energies where the measured $N_{c h} \nu_{+-, d y n}$ observed in central collisions appears more or less consistent with a resonance gas, but quite a bit stronger at LHC energy hinting at a stronger suppression of net charge fluctuations. The interpretation of these data is however complicated by diffusion and radial flow effects (Shuryak, 1998; Aziz and Gavin, 2004).
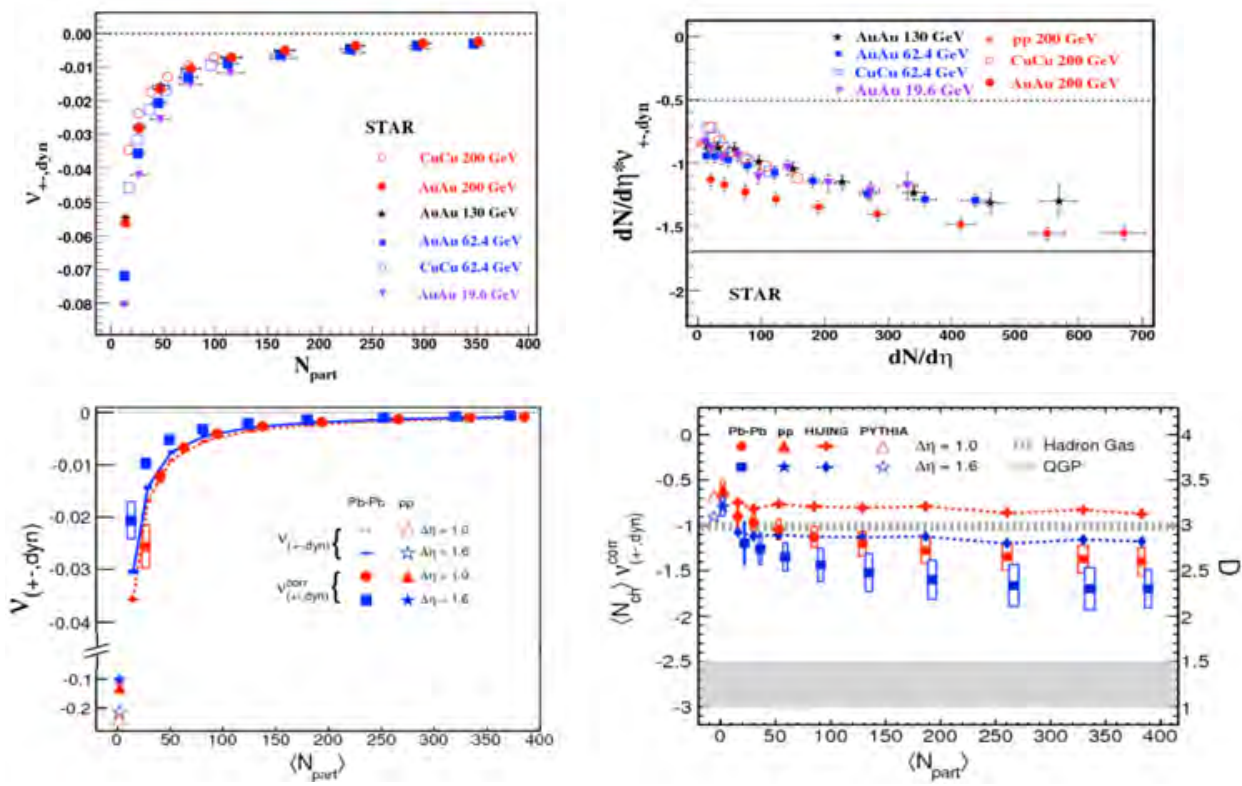

Fig. 2: Net charge fluctuations measurements by the STAR (Abelev et al., 2009a) and ALICE collaborations (Abelev et al., 2013)

\section{Balance Function}

The charge balance function is a differential correlation function closely related to measurements of net charge fluctuations. Pratt et al. proposed in (Bass et al., 2000; Jeon and Pratt, 2002) that a narrowing of the charge balance function, $B(\Delta \eta)$, measured as function of the pseudo rapidity acceptance, $\Delta \eta$, should 
signal delayed hadronization arising after the production of a "long-lived" QGP phase in relativistic heavy ion collisions.

$$
\begin{aligned}
B(\Delta \eta)= & \frac{\left\langle N_{-} N_{+}\right\rangle(\Delta \eta)}{\left\langle N_{-}\right\rangle}+\frac{\left\langle N_{+} N_{-}\right\rangle(\Delta \eta)}{\left\langle N_{+}\right\rangle} \\
& -\frac{\left\langle N_{+}\left(N_{+}-1\right)\right\rangle(\Delta \eta)}{\left\langle N_{+}\right\rangle}-\frac{\left\langle N_{-}\left(N_{-}-1\right)\right\rangle(\Delta \eta)}{\left\langle N_{-}\right\rangle}
\end{aligned}
$$

At relativistic energies, $\left\langle N_{-}\right\rangle \approx\left\langle N_{+}\right\rangle$, the balance function may then be expressed in term of integral correlators as follows:

$$
B(\Delta \eta)=\left\langle N_{c h}\right\rangle R_{+-}-\left\langle N_{+}\right\rangle R_{++}-\left\langle N_{-}\right\rangle R_{--}
$$

STAR(Adams et al., 2003b; Aggarwal et al., 2010) has measured the balance function as function of $\Delta \eta$ in a variety of collision systems at several beam energies and for both inclusive and specific particle species (e.g. pions, kaons, etc). It reported a substantial narrowing of the balance function in central $\mathrm{Au}+\mathrm{Au}$ collisions relative to widths measured in peripheral collisions, consistent with the notion of delayed hadronization. While radial flow may produce a slight narrowing of the balance function measured as function of $\Delta \eta$ (or $\Delta \phi$ ), the narrowing observed experimentally appears too strong to be explained solely on the basis of radial flow and is thus suggestive of the formation of a deconfined system with delayed particle production. ALICE (Abelev et al., 2013) carried out similar measurements in the $\mathrm{Pb}+\mathrm{Pb}$ system and obtained similar conclusions.
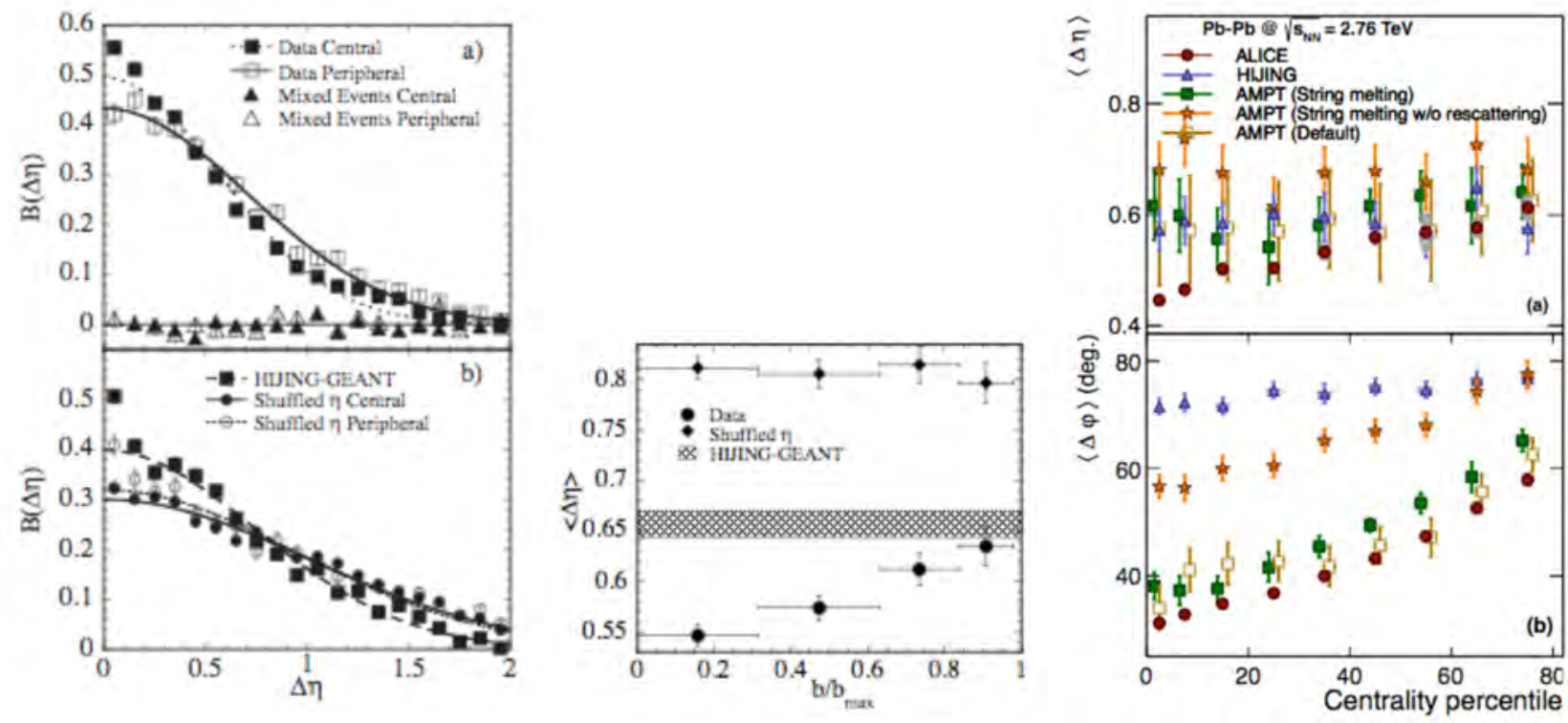

Fig. 3: Narrowing of the balance function observed by STAR (Adams et al., 2003b) and ALICE (Abelev et al., 2013) 


\section{Higher Moments of Net Charge Fluctuations}

Theoretical studies suggest the phase diagram of nuclear matter may be rather complex. In particular, one expects that the transition from a hadron gas to a quark gluon plasma might be a cross over transition at very small net baryon density and a first order phase transition at large net baryon density. This thus suggests the existence of a critical point (CP) at the end of the first order phase boundary (Fodor and Katz, 2002; Stephanov, 2004, 2005; Fodor and Katz, 2004). The location of this CP is subject to much speculations and no theoretical consensus exists as to what its position might be. It might however be possible to locate the critical point experimentally based on the notion that the correlation length $\xi$ is expected to diverge near $\mathrm{CP}$ in an infinite and static system. The systems formed in high energy heavy ion collisions are obviously not static or infinite, but various studies (Stephanov, 2009; Hatta and Stephanov, 2003; Karsch and Redlich, 2011; Rajagopal and Wilczek, 1993; Athanasiou et al., 2010) indicate that it might nonetheless be possible to identify a noticeable increase in the correlation length through measurements of higher moments net charge fluctuations. The STAR collaboration has reported a measurement of the kurtosis of the net charge fluctuations produced in in $\mathrm{Au}+\mathrm{Au}$ collisions at several beam energies (Aggarwal et al., 2010; Luo et al., 2011; Tarnowsky et al., 2011). Measurements reported thus far indicate the kurtosis scaled by the variance is essentially independent of both collision centrality and beam energy. These measurements are unfortunately inconclusive because of limited statistics and issues involved in corrected for efficiency losses. It is also possible the RHIC beam energy scan has simply missed the relevant range of net baryon density. Better observational techniques and larger data samples over a wider span of beam energies are consequently needed before one decidedly concludes on the existence of a critical point through this line of investigation.

\section{K to $\pi$ Yield Ratio Fluctuations}

The observation of a relatively sharp peak in the ratio of kaon to pion average yields, $\left\langle K^{+}\right\rangle /\left\langle\pi^{+}\right\rangle$, by the NA49 collaboration near $\sqrt{s_{N N}}=7 \mathrm{GeV}$ generated a great deal of interest and theoretical studies. It was in particular suggested that this spike might signal the phase transition or perhaps even the presence of a critical point. The presence of a critical point should in principle engender abnormal fluctuations in the yield of kaons relative to the production of pions. Several measurements of fluctuations of ratio kaon to pion yields, $R=N_{K^{+}} / N_{\pi^{+}}$, were thus undertaken. NA49(Roland et al., 2004; Alt et al., 2009) reported results in terms of so called dynamical fluctuations consisting of the difference of the width of the $R$ distribution measured in actual events relative to that obtained with mixed events. STAR(Abelev et al., 2009b) based its analysis on the $\nu_{K, \pi, d y n}$ observable extended from measurements of net charge fluctuation measurements. Comparison of STAR and NA49 data, shown in Fig. 4, indicate the two data sets are difficult to reconcile, unfortunately. More robust data, with better kaon to pion separation, and much larger data samples are thus 
direly awaited.

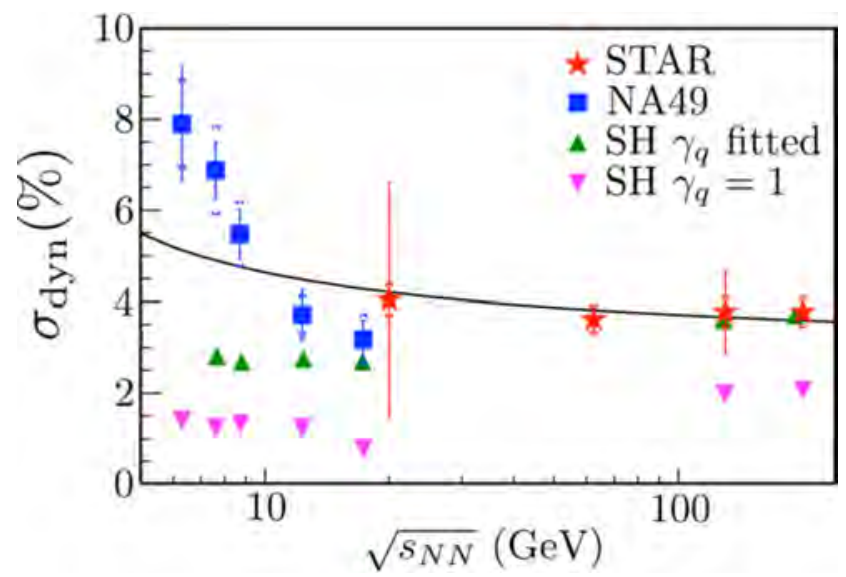

Fig. 4: Comparison of excess fluctuations of the kaon to pion fluctuations measured by the NA49 and STAR collaborations

\section{Transverse Momentum Fluctuations}

Transverse momentum fluctuations were suggested by Heiselberg (Heiselberg, 2001) and Stephanov et al. (Stephanov et al., 1998) as a probe of phase instabilities near the QCD vs. hadron phase boundary. These speculations have motivated several measurements by the NA49(Appleshauser et al., 1999). CERES (Adamova et al., 2003), STAR (Pruneau, 2005; Adams et al., 2005), PHENIX (Adcox et al., 2002b), and ALICE Collaborations. As for net charge and $K / \pi$ fluctuations, several distinct observables and methods can be used to measure transverse momentum fluctuations. Arguably, the simplest and most straightforward measurement method involves the $\left\langle\Delta p_{T} \Delta p_{T}\right\rangle$ observable first introduced by Voloshin (Voloshin et al., 1999).

$$
\left\langle\Delta p_{T} \Delta p_{T}\right\rangle=\frac{\int \rho_{2}\left(\vec{p}_{T, 1}, \vec{p}_{T, 2}\right) \Delta p_{T, 1} \Delta p_{T, 2} d p_{T, 1} d p_{T, 2}}{\int \rho_{2}\left(\vec{p}_{T, 1}, \vec{p}_{T, 2}\right) d p_{T, 1} d p_{T, 2}}
$$

where $\Delta p_{T, i} \equiv p_{T, i}-\left\langle p_{T}\right\rangle$, and $\left\langle p_{T}\right\rangle$ is the inclusive transverse momentum average of measured particles. By construction, the correlation function $\left\langle\Delta p_{T} \Delta p_{T}\right\rangle$ scales inversely to the number of correlated particle sources, it is thus meaningful to examine the product $d N / d \eta\left\langle\Delta p_{T} \Delta p_{T}\right\rangle$ as reported by the STAR collaboration(Pruneau, 2005; Adams et al., 2005). One finds that the scaled correlation strength has a very strong dependence on collision centrality thereby indicating that the systems formed in central collisions are considerably different than those produced in peripheral $\mathrm{Au}+\mathrm{Au}$ collisions or in proton - proton collisions. The radial flow model formulated by Voloshin (Voloshin, 2006) indicate the collision centrality dependence observed by STAR is largely due to radial flow effects. Measurements by ALICE (Heckel, 2011) of $\left\langle\Delta p_{T} \Delta p_{T}\right\rangle$ scaled by $p_{T}$, shown in Fig. 5, however suggest the correlation function is in fact suppressed 
in most central collisions relative to most peripheral collisions. Considerable work remains to disentangle effects of collective radial flow and those potentially associated with the proximity of a phase boundary.

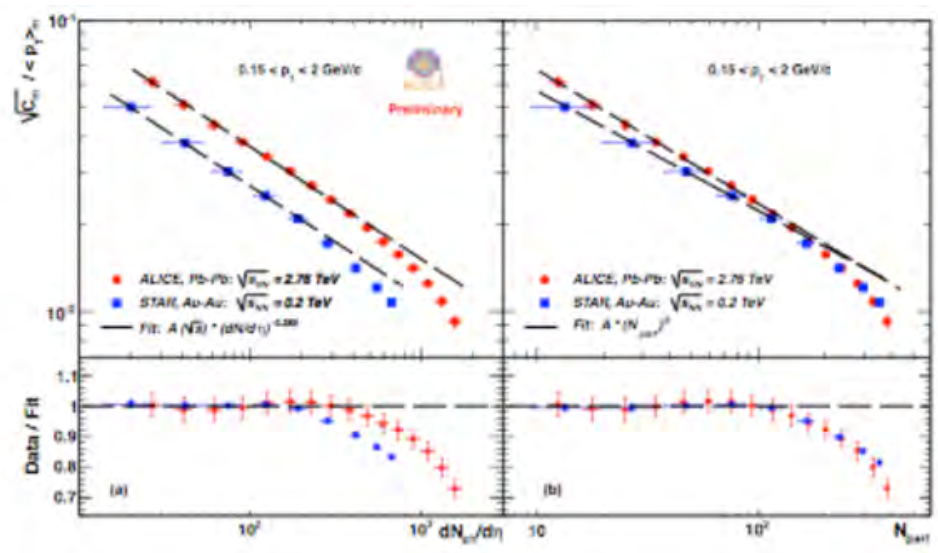

Fig. 5: Transverse Momentum Fluctuations Measured by the ALICE collaboration

\section{Conclusions}

Experiments at the CERN SPS, RHIC, and the LHC have conducted a wide range of correlation and fluctuation observables. While measurements of anisotropic flow coefficients lead to a rather unambiguous interpretation in terms of collective flow with low viscosity, considerably more work is yet required to reach a coherent picture based on measurements of fluctuation observables. Net charge fluctuation measured at RHIC and LHC show the systems produced in central collisions are clearly modified relative to those produced in peripheral or proton-proton collisions but the suppression of the fluctuations is not as large as that expected in the presence of a QGP phase. The suppression of the fluctuations is however larger at LHC possibly owing to the production of a more prominent QGP phase, smaller diffusion effects, or possibly more substantial radial flow effects. The identification of the QGP phase based on net charge fluctuation thus remains elusive. The observed narrowing of the balance function reported by both STAR and ALICE is, however, much more encouraging. Indeed, model calculations reported by Pratt indicate the observed narrowing cannot be explained on the basis of kinematic effects (radial flow) alone. The substantial narrowing observed is thus consistent with delayed hadronization, and the production of a deconfined QGP phase. Measurements of other fluctuations observables, such as higher moments of net charge, fluctuations of kaon vs pion yields, and transverse momentum fluctuations, though potentially promising, require further studies.

\section{References}

1. Abelev BI et al., (2009a) Beam-Energy and System-Size Dependence of Dynamical Net Charge Fluctuations Phys Rev C79 024906 
2. Abelev B et al., (2013) Net-Charge Fluctuations in $\mathrm{Pb}-\mathrm{Pb}$ collisions at $\sqrt{s_{N N}}=2.76 \mathrm{TeV}$ Phys Rev Lett 110 152301

3. B Abelev et al., (2013) Charge correlations using the balance function in $\mathrm{Pb}-\mathrm{Pb}$ collisions at $\sqrt{s_{N N}}=2.76 \mathrm{TeV}$ Phys Lett B723 267

4. Abelev BI et al., (2009b) K/pi Fluctuations at Relativistic Energies Phys Rev lett 103092301

5. Adamova D et al., (2003) Event by event fluctuations of the mean transverse momentum in 40, 80 and $158 \mathrm{~A}$ $\mathrm{GeV} / \mathrm{c} \mathrm{Pb}$ - Au collisions Nucl Phys A727 97

6. Adams J et al., (2003) Multiplicity fluctuations in Au+Au collisions at $\sqrt{s_{N N}}=130 \mathrm{GeV}$ Phys Rev $\mathbf{C 6 8} 044905$

7. Adams J et al., (2003) Narrowing of the balance function with centrality in au + au collisions at $\sqrt{s_{N N}}=130$ GeV Phys Rev Lett 9090

8. Adams J et al., (2005) Event by event $<p(t)>$ fluctuations in $\mathrm{Au}-\mathrm{Au}$ collisions at $\sqrt{s_{N N}}=130 \mathrm{GeV}$ Phys Rev C71 064906

9. Adcox K et al., (2002a) Net charge fluctuations in Au+Au interactions at $\sqrt{s_{N N}}=130 \mathrm{GeV}$ Phys Rev Lett 89 082301

10. Adcox A et al., (2002b) Event-by-event fluctuations in mean $\mathrm{p}(\mathrm{T})$ and mean $\mathrm{e}(\mathrm{T})$ in $\sqrt{s_{N N}}=130 \mathrm{GeV} \mathrm{Au}+\mathrm{Au}$ collisions Phys Rev C66 024901

11. Alt $\mathrm{C}$ et al., (2004) Electric charge fluctuations in central $\mathrm{Pb}+\mathrm{Pb}$ collisions at $20 \mathrm{~A} \mathrm{GeV}, 30 \mathrm{~A} \mathrm{GeV}, 40 \mathrm{~A} \mathrm{GeV}$, $80 \mathrm{~A} \mathrm{GeV}$, and $158 \mathrm{~A} \mathrm{GeV}$ Phys Rev C70 064903

12. Alt $\mathrm{C}$ et al., (2009) Energy dependence of particle ratio fluctuations in central $\mathrm{Pb}+\mathrm{Pb}$ collisions from $\mathrm{s}(\mathrm{NN}) * *(1 / 2)$ $=6.3 \mathrm{GeV}$ to $17.3 \mathrm{GeV}$ Phys Rev $\mathbf{C 7 9} 044910$

13. Aggarwal MM et al., (2010) Balance Functions from $\mathrm{Au}+\mathrm{Au}, \mathrm{d}+\mathrm{Au}$, and $\mathrm{p}+\mathrm{p}$ Collisions at $\sqrt{s_{N N}}=200 \mathrm{GeV}$ Phys Rev C82 024905

14. Aggarwal et al., (2010) Higher Moments of Net-proton Multiplicity Distributions at RHIC Phys Rev lett 105 022302

15. Appelshauser $\mathrm{H}$ et al., (1999) Event-by-event fluctuations of average transverse momentum in central $\mathrm{Pb}+\mathrm{Pb}$ collisions at $158 \mathrm{GeV}$ per nucleon Phys Lett B459 679

16. Asakawa M, Heinz U and Muller B (2000) Fluctuation probes of quark deconfinement Phys Rev Lett 852072

17. Athanasiou C, Rajagopal K and Stephanov M A (2010) Using Higher Moments of Fluctuations and their Ratios in the Search for the QCD Critical Point Phys Rev D82 074008

18. Aziz MA and Gavin S (2004) Causal diffusion and the survival of charge fluctuations in nuclear collisions Phys Rev C70 034905

19. Bass SA, Danielewicz P and Pratt S (2000) Clocking hadronization in relativistic heavy ion collisions with balance functions Phys Rev Lett $\mathbf{8 5} 2689$

20. Baym G and Heiselberg H (1999) Event-by-event fluctuations in ultrarelativistic heavy ion collisions Phys Lett B469 7

21. Bialas A (2002) Charge fluctuations in a quark anti-quark system Phys Lett $\mathbf{B 5 3 2} 249$

22. Bleicher M, Jeon S and Koch V (2000) Event-by-event fluctuations of the charged particle ratio from nonequi- 
librium transport theory Phys Rev C62 061902

23. Bopp FW and Ranft J (2001) Charged particle fluctuation as signal of the dynamics in heavy ion processes Eur Phys $J$ C22 171

24. Fodor $\mathrm{Z}$ and Katz SD (2004) Critical point of QCD at finite $\mathrm{T}$ and $\mu$, lattice results for physical quark masses JHEP 04050

25. Fodor Z and Katz SD (2002) Lattice determination of the critical point of QCD at finite $\mathrm{T}$ and mu JHEP $\mathbf{0 2} 014$

26. Hatta Y and Stephanov MA (2003) Proton number fluctuation as a signal of the QCD critical endpoint Phys Rev lett 91102003

27. Heckel S (2011) Event-by-event mean $p_{T}$ fluctuations in pp and $\mathrm{Pb}-\mathrm{Pb}$ collisions measured by the ALICE experiment at the LHC J Phys G38 124095

28. Heiselberg H (2001) Event-by-event physics in relativistic heavy ion collisions Phys Rept 351161

29. Jeon S and Koch V (1999) Fluctuations of particle ratios and the abundance of hadronic resonances Phys Rev Lett 835435

30. Jeon S and Koch V (2000) Charged particle ratio fluctuation as a signal for QGP Phys Rev Lett $\mathbf{8 5} 2076$

31. Jeon S and Pratt S (2002) Balance functions, correlations, charge fluctuations and interferometry Phys Rev $\mathbf{C 6 5}$ 044902

32. Karsch K and Redlich K (2011) Probing freeze-out conditions in heavy ion collisions with moments of charge fluctuations Phys Lett $\mathbf{B 6 9 5} 136$

33. Koch V, Bleicher M and Jeon S (2002) Event-by-event fluctuations and the QGP Nucl Phys A698 261

34. X Luo et al., (2011) Probing the QCD Critical Point with Higher Moments of Net-proton Multiplicity Distributions J Phys (Conference Series) 316012003

35. Pratt S (2002) Balance functions: A signal of late-stage hadronization Nucl Phys $\mathbf{A 6 9 8} 531$

36. Pruneau CA (2005) Excitation function of $<p_{t}>$ and net charge fluctuations at RHIC Acta Phys Hung A24 (2005) 85

37. Pruneau C, Gavin S and Voloshin S (2003) Net charge dynamic fluctuations Nucl Phys A715 661

38. Rajagopal K and Wilczek F (1993) Static and dynamic critical phenomena at a second order QCD phase transition Nucl Phys B399 395

39. Roland $\mathrm{C}$ et al., (2004) Event by event fluctuations of particle ratios in central $\mathrm{Pb}+\mathrm{Pb}$ collisions at $20 \mathrm{AGeV}$ to 158 AGeV J Phys G30 S1381

40. Shuryak EV (1998) Event per event analysis of heavy ion collisions and thermodynamical fluctuations Phys Lett B423 9

41. Shuryak EV and Stephanov MA (2001) When can long range charge fluctuations serve as a QGP signal? Phys Rev $\mathbf{C 6 3} 064903$

42. Stephanov MA (2004) QCD phase diagram and the critical point Prog Theor Phys Suppl 153139

43. Stephanov MA (2005) QCD phase diagram and the critical point Int J Mod Phys A20 4387

44. Stephanov MA (2009) Non-Gaussian fluctuations near the QCD critical point Phys Rev lett 102032301

45. Stephanov MA, Rajagopal K and Shuryak EV (1998) Signatures of the tricritical point in QCD Phys Rev Lett 
814816

46. Tarnowsky TJ et al., (2011) Searching for the QCD Critical Point Using Particle Ratio Fluctuations and Higher Moments of Multiplicity Distributions J Phys G38 124054

47. Voloshin SA, Koch V and Ritter HG (1999) Event-by-event fluctuations in collective quantities Phys Rev C60 024901

48. Voloshin SA (2006) Transverse radial expansion in nuclear collisions and two particle correlations Phys Lett B632 490

49. Zhang QH, Pop VT, Jeon S and Gale C (2002) Charged particle ratio fluctuations and microscopic models of nuclear collisions Phys Rev $\mathbf{C 6 6} 014909$. 